\title{
Novel Splice-Site and Missense Variant of PNPLA6 in an Austrian Family Causing Spastic Paraplegia-39 with Cerebellar Oculomotor Disorder
}

Sebastian Viertauer ( $\sim$ sebastian.viertauer@kepleruniklinikum.at)

Kepler Universitatsklinikum Neuromed Campus https://orcid.org/0000-0001-7497-3444

Ingo Kurth

Uniklinik RWTH Aachen: Universitatsklinikum Aachen

\section{Katja Eggermann}

Uniklinik RWTH Aachen: Universitatsklinikum Aachen

Christian Eggers

Kepler Universitatsklinikum Neuromed Campus https://orcid.org/0000-0002-4232-8624

\section{Research Article}

Keywords: motor neuron disease, hereditary spastic paraplegia, PNPLA6, novel genotype, novel phenotype,

Posted Date: January 5th, 2022

DOI: https://doi.org/10.21203/rs.3.rs-1142999/v1

License: @ (1) This work is licensed under a Creative Commons Attribution 4.0 International License. Read Full License 


\section{Abstract}

Background The term hereditary spastic paraplegia comprises an ever-expanding array of neurological disorders with distinct aetiologies. Spastic paraplegia 39 is one of the many variants with additional features of other organs and neurological systems. We describe a large kindred with two hitherto undescribed mutations of PNPLA6 and a novel clinical phenotype as, in addition to spastic paraplegia, affected subjects suffered from a prominent cerebellar oculomotor dysfunction.

Methods Three of five genetically tested family members of a large kindred were affected by spastic gait and cerebellar oculomotor dysfunction. Clinical, imaging, laboratory and electrophysiological data were analyzed. Genetic analysis was done using next-generation sequencing. Segregation analyses were performed by Sanger sequencing. To assess the pathogenicity of genetic variants on the encoded protein, in silico assessments were carried out.

Results Two hitherto unknown sequence variants in the PNPLA6 gene, a splice-site variant c.1635+3G>T and a missense variant c.3401A $>$ T, p.(Asp1134Val), were detected. Compound-heterozygous siblings presented with lower limb spasticity and a marked cerebellar oculomotor disorder accompanied by moderate hypogonadotropic hypogonadism in the female. A paternal uncle being homozygous for the splice-site variant of PNPLA6 presented with increased lower limb reflexes, an unstable gait and cerebellar oculomotor dysfunction. Treatment with 4-aminopyridin, a potassium channel blocker, lead to meaningful improvement of clinical symptoms.

Conclusion PNPLA6 gene variants are associated with a broad phenotypic spectrum to which we add cerebellar oculomotor dysfunction. In our kindred, the full clinical manifestation only occurred in compound-heterozygous subjects indicating that biallelic pathogenic variants lead to more serious and earlier onset of symptoms. Our findings emphasize the role of PNPLA6 in different neurodegenerative disorders.

\section{Introduction}

Hereditary spastic paraplegias (HSPs), also known as Strumpell-Lorrain disease, are a heterogeneous group of monogenetic neurodegenerative disorders where the unifying clinical presentation is that of progressive spasticity and paraplegia.(1) Inheritance follows autosomal-dominant (AD), autosomal-recessive (AR) or X-linked patterns. The classification is based on the affected genes. $(2,3)$ So far more than 80 causal genes or loci have been detected and resulted in a spastic paraplegia gene (SPG) classification. $(4,5)$ The histopathological feature of HSPs is a length-dependent axonal degeneration of motor and sensory fibers within the corticospinal tract and the dorsal column. $(6,7)$

Spastic Paraplegia 39 (SPG39), an AR SPG, was first described in 2008 by Rainier et al.(8) and is characterized by a childhood onset of progressive spasticity of the lower limbs and progressive weakness of the upper and lower limb muscles.

The phenotype of SPG39 corresponds to SPG20, an AR form of HSP with lower motor neuron involvement, and to organophosphorous compound-induced delayed neuropathy (OPIDN) with distal axonal degenerations.(8-10) SPG39 is caused by mutations in PNPLA6, a gene encoding neuropathy target esterase (NTE). Interestingly, mutations of PNPLA6 have also been found to cause other rare hereditary neurodegenerative diseases such as Gordon-Holmes, Boucher-Neuhauser, Laurence-Moon and Oliver-McFarlane syndrome.(11-13)

Rainier et al.(8) reported two families in which affected subjects developed a childhood onset progressive spastic weakness of lower limbs and wasting of distal upper and lower limb muscles. In the consanguineous family, affected subjects carried a homozygous mutation in the PNPLA6 gene c.3034A > G, p.(M1012V) that was shown to alter the catalytic domain of the encoded protein NTE. Moreover, in the non-consanguineous family affected subjects were compound heterozygotes where one allele had a missense mutation c.2669G $>$ A, p. (R890H) and the other allele had an insertion mutation c.2946_2947insCAGC, p.(S982fs1019). Both variants cause an impaired enzymatic function of NTE.

In 2014, Synofzik et al.(11) reported a family with HSP harboring the compound heterozygous variants c.787G>A, p.(Val263lle) and c.2519G>A, p.(Gly840Glu) in the PNPLA6 gene. Affected subjects presented with spasticity, hyperreflexia of lower extremities, positive Babinski's sign and a mild motor neuropathy.

Yoon et al.(14) presented a sole patient with a pure spastic paraplegia phenotype characterized by motor delay and gait abnormalities with falls. This patient was heterozygous for the variant c.2944_2947dup, p.(Arg983ArgfsX86) of the PNPLA6 gene. 
We here describe a large kindred with two hitherto undescribed mutations in the PNPLA6 gene. Their clinical phenotype was novel as, in addition to spastic paraplegia, they had a marked cerebellar oculomotor dysfunction.

\section{Case Presentation}

S1 (Subject 1), the index subject, was a 29-year-old male presenting with benign peripheral paroxysmal vertigo. Routine neurologic examination revealed marked gait disturbance for which the patient had never sought medical advice. He reported normal milestones during childhood and worked as a skilled specialist in manufacturing.

On examination he had grossly spastic gait with normal Romberg test and brisk muscle stretch reflexes with sustained ankle clonus. However, Babinski's sign was negative. His muscles were neither weak nor atrophic. Oculomotor testing revealed massive saccadic pursuit movements. There was coarse nystagmus evoked by upward and horizontal gaze. On downward gaze, however, the direction of the nystagmus was upward (to his forehead) (Additional file 2; Additional file 3). There was no nystagmus in primary position. Rebound nystagmus could easily be elicited. HIT (head impulse test) according to Halmagyi showed normal VOR (vestibular ocular reflexes), but the suppression of the VOR by fixation was markedly impaired. Optokinetic nystagmus was reduced. Despite these prominent findings the patient had no subjective visual complaints. There was no ataxia of gait, the upper limbs or of speech. The remainder of the neurologic examination was inconspicuous. In particular there was no sensory, autonomic or cognitive dysfunction.

In S2 (subject 2), his 35-year-old sister, an office employee with good cognitive abilities, a marked gait disturbance had been present from early childhood with a very slowly progressive course. In terms of quality, her neurological findings were identical to subject S1, however, somewhat more pronounced. At the age of around 18 years, she developed secondary amenorrhea.

The father, S5 (subject 5), of S1 and S2 had no complaints. On examination he had sustained ankle clonus without Babinski's sign. Gait and balance were normal and there was no oculomotor disturbance.

The same was true for the mother, S4 (subject 4), in whom ankle clonus was present but exhaustible.

S3 (subject 3), the 71-year-old paternal uncle of S1 and S2, became symptomatic only in his early fourties with gait disturbance and vertigo on head movement. On examination there was gaze evoked nystagmus to both sides and upwards but not downwards, saccadic pursuit movements, moderately impaired VOR and impairment of fixation-induced suppression of the VOR (corresponding with impaired reading on smooth sinusoid head movements).

Gait was clumsy but not spastic or atactic; Romberg test was normal. Muscle strength was normal without any atrophy, but knee and ankle jerks were markedly increased with sustained ankle clonus. Babinski's sign was negative. There were no sensory findings.

The son of S3 had no complaints and was normal on thorough neurological examination.

In the extended family one female cousin in the paternal line of S1 and S2 had died from bulbar-onset amyotrophic lateral sclerosis at the age of 33 , and another cousin had a well-founded diagnosis of multiple sclerosis. Genetic material was not available from these two subjects.

\section{Paraclinical Findings}

Routine blood tests were done in S1, S2 and S3 and did not yield any systematic abnormalities. Hormone status was compatible with hypogonadotropic hypogonadism in S1 and S2.

In the CSF, taken from S1, routine parameters as well as neurofilament light, total tau and phosphorylated tau were unrevealing.

In S1 and S3, standard MRI (Siemens Avanto; 1,5 Tesla) of the brain and the entire spinal cord was normal without cerebellar or spinal cord atrophy. MRI or other radiological examinations were not available from the other family members.

In VNG (videonystagmography) of S1 and S2 the most prominent finding was pathologic fixation suppression of the VOR (Fig. 2). Nerve conduction studies, done in S1 and S2, were conspicuous only for increased F-latencies in the peroneal and tibial nerves of only S1. Needle EMG was normal. In S2, magnetic evoked potentials showed increased central motor conduction times to the distal legs while there were no responses in S1.

Page 3/9 
Ophthalmologic examination, done in S2, was normal including OCT (optical coherence tomography). Degree of relationship, genotype and clinical phenotype of subjects are given in Table 1 (more detailed information Additional file 2).

\section{Materials And Methods}

The study was approved by the local ethics committee, and subjects provided written informed consent (Declaration of Helsinki).

An Illumina Enrichment Kit (Panel-ID 109980) was used to capture the genes under investigation. The generated library was sequenced on a MiSeq sequencer (Illumina). The generation of the analyzed "bam" or "FastQ" files was carried out with the MiSeqReporter Software (v.2.6.2, Illumina, reference genome: hg19).

The generated raw data were analyzed using the SeqNext module of the SeqPatient software (JSI, v.4.3.0-B503).

Variants with a frequency of the rarer allele of $>1 \%$ in relevant databases (EXAC, dbSNP, 1000G), synonymous variants and variants that are not within the coding region, outside of the canonical splice sites, as well as variants which are not bi-directionally covered were not considered.

The following genes were analyzed using the Illumina Enrichment Kit:

ATL1 (SPG3), SPAST (SPG4), CYP7B1 (SPG5), PGN(SPG7), KIF5A (SPG10), SPG11 (SPG11), ZFYVE26 (SPG15), REEP1 (SPG31), ACP33, AMPD2, AP4B1, AP4E1, AP4M1, AP4S1, AP5Z1, ARL6IP1, ARSI, B4GALNT1, C120RF65, C19ORF12, CAPN1, CYP2U1, DDHD1, DDHD2, ENTPD1, ERLIN1, ERLIN2, FA2H, GAD1, GBA2, GJC2, HSPD1, KCNA2, KIAA0196, KIAA1840, KIF1C, KLC2, L1CAM, NIPA1, NT5C2, PGAP1, PLP1, PNPLA6, RAB3GAP2, REEP2, RTN2, SLC16A2, SLC33A1, SPG20, USP8, VPS37A, WDR48, ZFR, ZFYVE27

Coverage was $>30 \mathrm{X}$ for app. $99 \%$ of the regions of interest.

For segregation analyses of the variants, the relevant sequences of the PNPLA6 gene were PCR-amplified and subsequently sequenced using standard Sanger sequencing protocols. To exclude copy number variants in REEP1, SPG7, SPAST and ATL 1, a semi-quantitative MPLA (Multiplex Ligation Dependent Probe Amplification) method (Kits no. P231-B2 and P165-C2, MRC-Holland) was used.

For bioinformatic assessment of pathogenicity Alamut v.2.15.0 and PolyPhen-2 were used. It must be taken into account that these programs only provide information on possible pathogenicity. The pathogenicity assessment of genomic variants is essentially based on the consensus recommendations of the ACMG (American College of Medical Genetics and Genomics).(15)

\section{Results}

In S2, NGS (next generation sequencing) identified two variants in the PNPLA6 gene (OMIM: 603197; NM_001166111): a missense mutation c.3401A>T, p.(Asp1134Val) and a splice-site variation c.1635+3G>T possibly influencing the donor splice-site of intron 15 . To date, neither of these sequence alterations have been described in the literature or in databases. The missense variant c.3401A>T in exon 29 is non-synonymous, i.e. it leads to the change of the amino acid Asp (aspartate) to Val (valine), and it affects a highly conserved part of the catalytic centre of the NTE.

Bioinformatics and in silico assessments by online tools showed a high likelihood of a deleterious effect of this mutation on the structure and function of its protein.

The substitution in intron 15 might lead to aberrant splicing according to splice-site prediction tools. No further pathogenic or possibly pathogenic variants were detected. MLPA did not show any deletions or duplications of single exons of REEP1, SPG7, SPAST and ATL 1 .

Sanger sequencing confirmed heterozygosity for each of the detected variants in patients S2 and S1 (Additional file 1A, Additional file 1B). Segregation analysis revealed compound-heterozygosity and indicated AR inheritance. S4 showed heterozygosity for the missense variant c.3401A>T, p.(Asp1134Val) assuming her as heterozygous carrier of the possibly disease-causing sequence alteration in PNPLA6 gene, whereas S5 and S3 were homozygous carriers of the splice-site variant c.1635+3G >T. Partial family pedigree is given in Figure 1 .

(Fig. 1)

Taken together, the clinical phenotype of siblings S1 and S2, who shared the same mutations in PNPLA6 gene (c.1635+3G>T/c.3401A>T, p.Asp1134Val) as a compound heterozygosity, consisted of lower limb spastic paraparesis and a marked cerebellar oculomotor disorder accompanied by hypogonadotropic hypogonadism. In contrast, their father, a homozygous carrier of the PNPLA6 variant c.1635+3G $>T$, 
was free of complaints but had sustained ankle clonus. His older brother S3 with the same homozygous mutation had gait disturbance with increased stretch reflexes and an oculomotor dysfunction of the cerebellar type. The mother S4, a heterozygous carrier of c.3401A>T, p.(Asp1134Val) was free of complaints but she had a somewhat brisk ankle reflex without sustained clonus. Oculomotor function in both parents was normal.

4-aminopyridine 10mg t.i.d (ter in die) was given to subjects S1, S2 and S3 who requested symptomatic treatment. All reported meaningful improvement in dexterity and steadiness of gait. Improvement in cerebellar oculomotor function was documented in S2 where VNG revealed coarse saccadic eye movements on testing the fixation-induced suppression of the VOR when the patient was off the drug and visible improvement one hour after 10mg 4-Aminopyridin (Fig. 2).

Table 1

Clinical and molecular findings of subjects

\begin{tabular}{|c|c|c|c|c|c|c|c|c|c|}
\hline Subject & Sex & Age & $\begin{array}{l}\text { Relative } \\
\text { to index } \\
\text { subject }\end{array}$ & Mutation & Zygosity & $\begin{array}{l}\text { Increased } \\
\text { lower } \\
\text { limb } \\
\text { reflexes; } \\
\text { sustained } \\
\text { ankle } \\
\text { clonus c }\end{array}$ & $\begin{array}{l}\text { Gait } \\
\text { abnormality }\end{array}$ & $\begin{array}{l}\text { Cerebellar } \\
\text { oculomotor } \\
\text { disturbance }\end{array}$ & $\begin{array}{l}\text { Hypogonadotropic } \\
\text { hypogonadism }\end{array}$ \\
\hline S1 & $\mathrm{m}^{\mathrm{b}}$ & 29 & $\begin{array}{l}\text { Index } \\
\text { subject }\end{array}$ & $\begin{array}{l}\text { c. } 1635+3 G>T \\
\text { c. } 3401 A>T\end{array}$ & $\begin{array}{l}\text { Compound } \\
\text { heterozygous }\end{array}$ & $\begin{array}{l}\text { Yes } \\
\text { (marked) }\end{array}$ & Yes & $\begin{array}{l}\text { Yes } \\
\text { (marked) }\end{array}$ & Yes \\
\hline S2 & $f^{a}$ & 35 & Sister & $\begin{array}{l}\text { c. } 1635+3 G>T \\
\text { c. } 3401 A>T\end{array}$ & $\begin{array}{l}\text { Compound } \\
\text { heterozygous }\end{array}$ & $\begin{array}{l}\text { Yes } \\
\text { (marked) }\end{array}$ & Yes & $\begin{array}{l}\text { Yes } \\
\text { (marked) }\end{array}$ & Yes \\
\hline S3 & $\mathrm{m}$ & 71 & $\begin{array}{l}\text { Paternal } \\
\text { uncle }\end{array}$ & c. $1635+3 G>T$ & Homozygous & $\begin{array}{l}\text { Yes } \\
\text { (marked) }\end{array}$ & Yes & $\begin{array}{l}\text { Yes } \\
\text { (marked) }\end{array}$ & None \\
\hline S4 & $f$ & 55 & Mother & c. $3401 \mathrm{~A}>\mathrm{T}$ & Heterozygous & $\begin{array}{l}\text { Yes } \\
\text { (marked) }\end{array}$ & None & None & None \\
\hline S5 & $\mathrm{m}$ & 58 & Father & c. $1635+3 G>T$ & Homozygous & $\begin{array}{l}\text { Yes } \\
\text { (marked) }\end{array}$ & None & None & None \\
\hline
\end{tabular}

a female

b male

c Tendon reflexes were graded by a reference scale from the National Institute of Neurological Disorders and Stroke (NINDS).

\section{Discussion}

Among the more than 80 different genetic causes of HSP, SPG39, which is caused by alterations in the gene PNPLA6, is likely to be one of the rarest forms. While PNPLA6 has been implicated in a variety of disorders, among which the leading manifestations are often extraneural, spastic paraplegia as the leading or even sole manifestation has been described in only three case reports. PNPLA6 causes different neurodegenerative motor diseases with a wide spectrum of symptoms. Rainier et al.(8) postulated that complex HSP, i.e. further clinical features in addition to spasticity such as distal muscle wasting, is the most common phenotype of $P N P L A 6$ mutations. However, Synofzik et al.(11) showed that besides complex HSP which is "the peak of the iceberg", PNPLA6 can cause a spectrum of neurodegenerative disorders with four main clinical features: ataxia, chorioretinal dystrophy, spasticity and hypogonadism. So far cerebellar oculomotor dysfunction has not been associated with PNPLA6 mutations.

The family reported here has a novel clinical phenotype due to novel compound heterozygous mutations in PNPLA6. Apart from lower limb spasticity with spastic gait, the most salient feature was marked oculomotor dysfunction comprising saccadic pursuit movements, gaze evoked nystagmus, rebound nystagmus and impaired suppression of the VOR by fixation. Most peculiar, however, was an intermittent variant of downbeat nystagmus where on downward gaze there was a nystagmus beating upwards (to the forehead). While these findings are typical for cerebellar dysfunction other cerebellar features such as gait ataxia and ataxia of upper limbs or speech were conspicuously absent. As the sole non-neurologic feature, patient S1 \& S2 had low concentrations of blood gonadotropic hormones, indicating mild hypogonadotropic hypogonadism. Taken together, the organ systems affected to the extent of becoming clinically apparent were the cortical motor neurons, cerebellar neurons or connections and, to a much lesser extent, the reproductive endocrine 
system. Hufnagel et al.(13) found individual tissues to express different levels of NTE activity. Thus, in our patients, NTE activity was likely most prominently reduced in cortical motor and cerebellar neurons.

Pathogenic mutations of PNPLA6 hitherto described all affect genetic sequences that code for the catalytic domain of NTE and that are highly conserved across species. There are a variety of PNPLA6 phenotypes with specific combinations of clinical features such as spastic ataxia (spasticity \& ataxia), Gordon Holmes syndrome (spasticity, primarily gait ataxia \& hypogonadism), Boucher-Neuhäuser syndrome (ataxia, hypogonadism \& chorioretinal dystrophy) and pure HSP (spasticity).(11) With an upper motor neuron disorder, isolated oculomotor cerebellar dysfunction and a limited degree of hypogonadism we add a novel phenotype to the spectrum of $P N P L A 6-$ associated disorders.

In our subjects S1 and S2 with compound heterozygosity the onset was earlier and the clinical symptoms more severe than in their father S5 and uncle S3 who both were homozygous for the splice-site variant c. $1635+3 \mathrm{G}>\mathrm{T}$. This suggests a more deleterious impact of the missense variant c.3401A>T than of the splice-site variant c.1635+3G $>$ T. Although S3 and S5 carried the same novel splice-site variant the older brother S3, with cerebellar dysfunction and spasticity, was clinically more affected than his younger brother S5 who only had brisk reflexes. This finding corresponds to previous findings of Synofzik et al.(11) who found weak genotype-phenotype correlations. Although S3 was 13 years older than S5 he had become symptomatic already at the age of 40, when he was 18 years younger than S5 who still had neither a cerebellar nor gait disturbance. A further possible explanation for the discrepancy between genotype and phenotype in S3 and S5 might be epigenetic factors influencing the splicing process of PNPLA6 gene. Our findings mirror those of previous studies where the clinical phenotype was found to be neither dependent on the location within the gene nor the type of the pathogenic variant. $(11,13,16)$ Hence, a genotype-phenotype correlation between our novel PNPLA6 variants and clinical phenotypes cannot be postulated.

Subjects with NTE mutations frequently suffer from progressive upper- more than lower-motor neuron disease leading to the assumption that NTE plays a major role in the integrity of motor axons.(8) Amyotrophic lateral sclerosis is a similar progressive degenerative motor neuron disorder. Rainier et al.(8) considered mutations of the NTE to contribute to the pathogenesis of amyotrophic lateral sclerosis. Interestingly, a cousin of subject S1 and S2 from the paternal line died of bulbar-onset amyotrophic lateral sclerosis at the age of 33. This raises speculations that her amyotrophic lateral sclerosis was based on a PNPLA6 mutation; however, she did not undergo genetic testing.

4-aminopyridine, a potassium channel blocker that is being used in downbeat nystagmus syndrome, a similar disorder of cerebellar nuclei and connections, proved to be clinically beneficial in our patients.

\section{Conclusion}

To summarize, our observations expand the already wide spectrum of clinical phenotypes reported with alterations in the $P N P L A 6$ gene. The hitherto undescribed (compound heterozygous) genotype was associated with a novel phenotype comprising prominent dysfunction of cerebellar oculomotor structures and the upper motor neuron system as well as some degree of hypogonadotropic hypogonadism.

\section{Abbreviations}

$\mathrm{AD}=$ autosomal-dominant; $\mathrm{AR}=$ autosomal-recessive; $\mathrm{HIT}=$ head impuls test; $\mathrm{HSP}=$ hereditary spastic paraplegia; $\mathrm{MLPA}=\mathrm{Multiplex}$ Ligation Dependent Probe Amplification; NTE = neuropathy target esterase; OCT = optical coherence tomography; OPIDN = organophosphorous compound-induced delayed neuropathy; SPG = spastic paraplegia gene; SPG39 = spastic paraplegia-39; VNG = videonystagmography; VOR = vestibular-ocular reflex;

\section{Declarations}

Ethics approval: This study was approved by the local ethics committee of Johannes Kepler University Linz (\#1105/2020).

Consent for publication: A consent form for publication was signed by each participant and forms are available up on request to the corresponding author. 
Availability of data and materials: All data generated or analysed during this study are included in this submitted/published article (and in its supplementary section). For further information please contact the corresponding author.

Additional material: Additional material is available at Orphanet Journal of Rare Diseases online.

Competing interests: The authors declare that they have no competing interests.

Funding statement: No funding was received towards this work.

Author's contributions: SV and CE clinically examined the subjects, done literature research, initiated further diagnostics and genetic analyses, started therapeutic attempt with 4-aminopyridine and were responsible for subjects follow-up; IK and KE done literature research, performed genetic analyses and wrote methods section. All authors read and approved the final manuscript.

Acknowledgements: No applicable.

\section{References}

1. Shribman S, Reid E, Crosby AH, Houlden H, Warner TT. Hereditary spastic paraplegia: from diagnosis to emerging therapeutic approaches. Lancet Neurol. 2019;18(12):1136-1146.

2. Harding AE. Hereditary" pure" spastic paraplegia: a clinical and genetic study of 22 families. J Neurol Neurosurg Psychiatry. 1981;44(10):871-883.

3. Harding AE. Classification of the hereditary ataxias and paraplegias. Lancet. 1983;321(8334):1151-1155.

4. Schüle R, Schöls L. Genetics of hereditary spastic paraplegias. In: Seminars in Neurology. Vol 31. @ Thieme Medical Publishers; 2011:484-493.

5. Schüle R, Wiethoff S, Martus P, et al. Hereditary spastic paraplegia: clinicogenetic lessons from 608 patients. Ann Neurol. 2016;79(4):646-658.

6. Harding AE. Hereditary spastic paraplegias. In: Seminars in Neurology. Vol 13.; 1993:333-336.

7. Fink JK. Advances in the hereditary spastic paraplegias. Exp Neurol. 2003;184:106-110.

8. Rainier S, Bui M, Mark E, et al. Neuropathy target esterase gene mutations cause motor neuron disease. Am J Hum Genet. 2008;82(3):780-785

9. Cross HE, McKusick VA. The Troyer syndrome: a recessive form of spastic paraplegia with distal muscle wasting. Arch Neurol. 1967;16(5):473-485.

10. Patel H, Cross H, Proukakis C, et al. SPG20 is mutated in Troyer syndrome, an hereditary spastic paraplegia. Nat Genet. 2002;31(4):347-348.

11. Synofzik M, Gonzalez MA, Lourenco CM, et al. PNPLA6 mutations cause Boucher-Neuhäuser and Gordon Holmes syndromes as part of a broad neurodegenerative spectrum. Brain. 2014;137(1):69-77.

12. Topaloglu AK, Lomniczi A, Kretzschmar D, et al. Loss-of-function mutations in PNPLA6 encoding neuropathy target esterase underlie pubertal failure and neurological deficits in Gordon Holmes syndrome. J Clin Endocrinol Metab. 2014;99(10):E2067-E2075.

13. Hufnagel RB, Arno G, Hein ND, et al. Neuropathy target esterase impairments cause Oliver-McFarlane and Laurence-Moon syndromes. J Med Genet. 2015;52(2):85-94.

14. Yoon G, Baskin B, Tarnopolsky M, et al. Autosomal recessive hereditary spastic paraplegia-clinical and genetic characteristics of a well-defined cohort. Neurogenetics. 2013;14(3):181-188.

15. Richards S, Aziz N, Bale S, et al. Standards and guidelines for the interpretation of sequence variants: a joint consensus recommendation of the American College of Medical Genetics and Genomics and the Association for Molecular Pathology. Genet Med. 2015;17(5):405-423.

16. Kmoch S, Majewski J, Ramamurthy V, et al. Mutations in PNPLA6 are linked to photoreceptor degeneration and various forms of childhood blindness. Nat Commun. 2015;6(1):1-10.

\section{Figures}




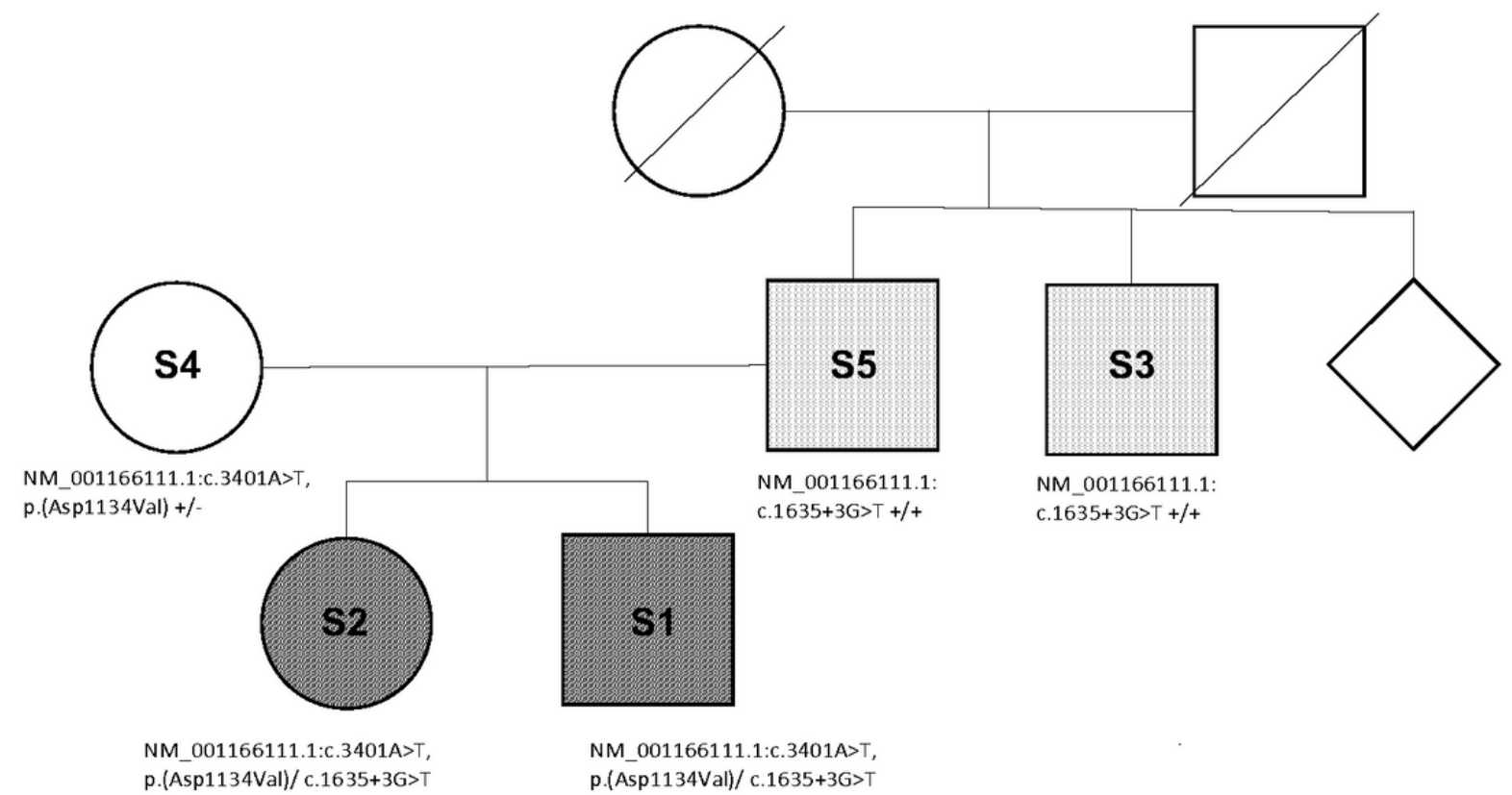

Figure 1

Partial pedigree of PNPLA6 family with novel genotype and phenotype. The pedigree structure of the PNPLA6 family; Affected subjects are shown as filled symbols. Compound-heterozygous subjects $(\mathrm{S} 1, \mathrm{~S} 2)$ presented with the novel genotype and phenotype consisting of a marked cerebellar oculomotor dysfunction, lower limb spasticity and a hypogonadotropic hypogonadism are shown in black. Grey symbols present subjects S3 and S5 suffering from a milder phenotype. 

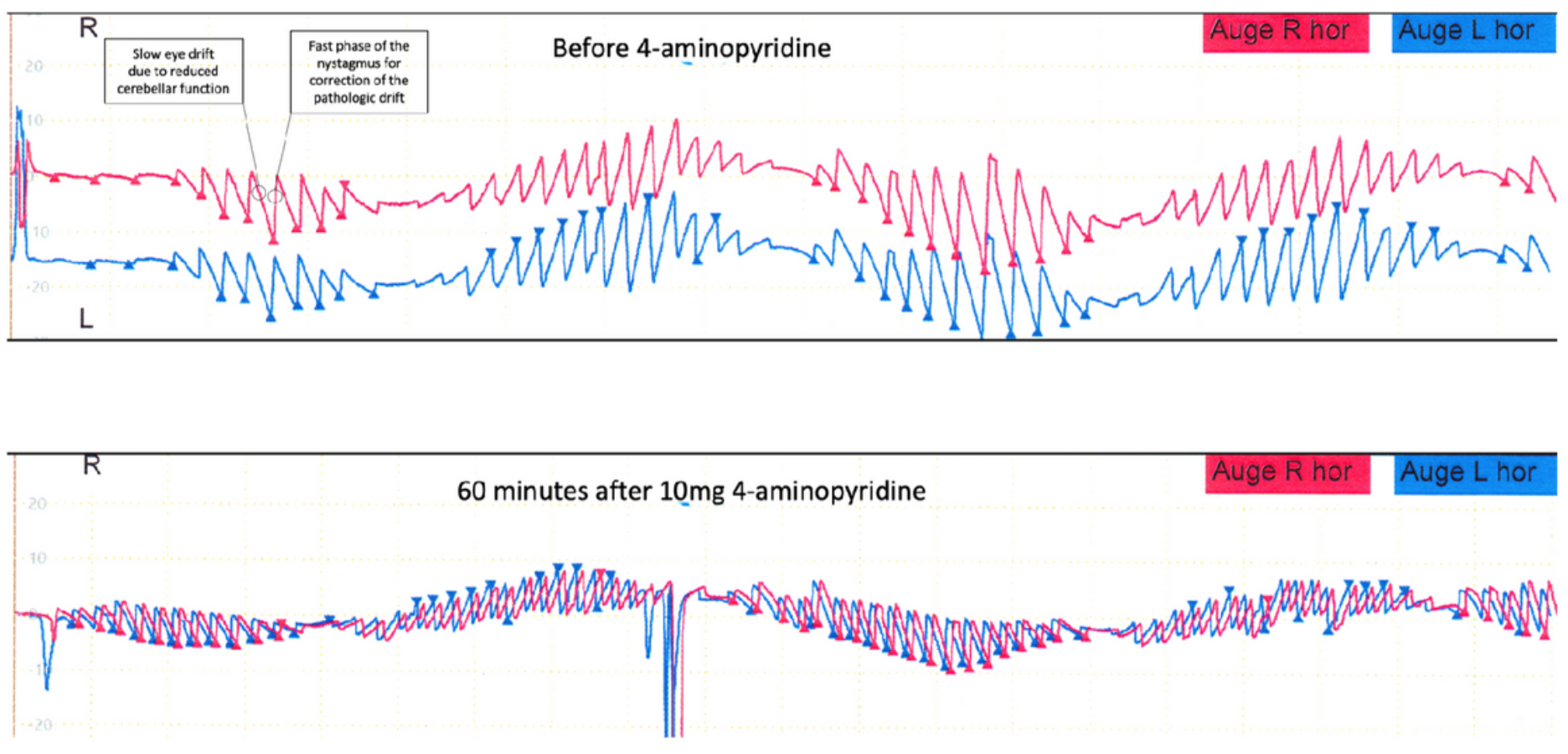

\section{Figure 2}

Suppression by fixation of the VOR before and after 4-aminopyridine in VNG. Suppression by fixation of the VOR, before and after 4aminopyridine. The seated subject is repeatedly turned around his vertical axis with sinusoidal movements while fixating a visual target. The visual target is moving in parallel with his body and head so that, in order to continuously fixate the target, the subject needs to keep his eyes in the primary position. Without 4-aminopyridine a high-amplitude drift with corresponding nystagmus is seen in both (horizontal) directions. 60 minutes after $10 \mathrm{mg}$ 4-aminopyridine the amplitude of drift and nystagmus is significantly reduced. The angular velocity and amplitude of rotation were kept the same in the two examinations.

\section{Supplementary Files}

This is a list of supplementary files associated with this preprint. Click to download.

- additionalfile1A.pdf

- additionalfile1B.pdf

- additionalfile2.pdf

- additionalfile3.mp4 